\title{
Visiones de Hollywood: la novela española como reflejo del debate cinematográfico de principios de siglo $\mathrm{XX}^{1}$
}

\author{
Patricia BARRERA VELASCO \\ Universidad Complutense de Madrid \\ pbarrera@ucm.es
}

\begin{abstract}
RESUMEN
Muchos de los autores españoles de la Edad de Plata sintieron fascinación por el cine y lo conocieron desde dentro: escribieron guiones, dirigieron películas y llegaron a actuar en alguna ocasión. Además, lo incluyeron como tema en sus obras literarias. Se estudian, aquí, algunos rasgos fundamentales de varias novelas del primer tercio del siglo XX, que muestran las reflexiones e inquietudes que suscitó el nuevo arte en los escritores del momento. A través de estos textos expondré algunas de las ideas más importantes que en aquellos años llenaron el debate en torno al cine, tales como la falsedad del mundo del cinema, la mitomanía creada en torno a los actores, las reflexiones sobre el público, las posibilidades estéticas del nuevo espectáculo y su comparación con el teatro. Casi todos ellos narran la llegada a Hollywood de un aspirante a actor y describen el ambiente de la gran urbe cinematográfica de los años veinte y treinta pero, en unos casos, desde la perspectiva de la novela de vanguardia y, en otros, desde los postulados de la novela social de preguerra.
\end{abstract}

Palabras clave: Hollywood, escritores, novela, cine, Edad de Plata.

${ }^{1}$ Este artículo se inscribe dentro de las actividades del grupo de investigación "Temas y Géneros de la Literatura Española de la Edad de Plata (y su proyección) ( $\mathrm{N}^{\circ}$ 941375), en el marco del Programa de Creación y Consolidación de Grupos de Investigación de la Universidad Complutense. Se trata de una breve muestra de una investigación más amplia, todavía en proceso, que formará parte de mi tesis doctoral. Por ello, ofrezco solo una síntesis del análisis de las novelas objeto de estudio. Quiero agradecer a mi compañera y amiga Cristina Arias Vegas sus sugerencias para el título de este trabajo. 


\begin{abstract}
Many of the Spanish authors of the Silver Age felt fascination for the cinema and knew it from the inside: they wrote scripts, directed films and they even acted occasionally. In addition, they included the cinema as a theme in their literary works. Some fundamental features of several novels of the first third of the 20th century are studied in this article, and they show the reflections and concerns that gave rise to this new art in the writers of the time. Through these texts, I shall explain some of the most important ideas that made debate over the cinema in those years, such as the falsity of the cinema, the mythomania created around the actors, the reflections on the public, the aesthetic possibilities of the new show and its comparison with the theatre. Almost all of them narrate the arrival in Hollywood of an aspiring actor and describe the environment of the metropolis of the cinema of the 1920s and 1930s. In some cases it is narrated from the perspective of the Vanguard novel and, in others, from the postulates of the pre-war social novel.
\end{abstract}

Keywords: Hollywood, writers, novel, cinema, Silver Age.

En el primer tercio del siglo XX, el debate sobre el cine ocupó cientos de páginas de la prensa española, que se convirtió en escenario idóneo para que, tanto periodistas como escritores, se situaran bien en el lado de los férreos defensores del cinema, bien en el de los detractores de un medio que consideraban solamente un entretenimiento sin visos artísticos. Los intelectuales españoles dejaron impresas sus opiniones, reflexiones y comentarios sobre el nuevo espectáculo en diarios ( $E l$ Liberal, La Libertad, ABC, Heraldo de Madrid, El Sol), revistas de información general (Nuevo Mundo, Blanco y Negro, La Esfera), revistas intelectuales (España, La Gaceta Literaria, Revista de Occidente) y revistas sobre cine (El Cine, La Pantalla, Popular Film, Nuestro Cinema). El crítico Rafael Utrera asegura que

el interés de nuestros intelectuales por el discurrir cinematográfico supone un proceso lento y muy diverso; (...). Los juicios y actitudes pro o anticinematográficos (...) es [sic] acorde con las manifestaciones y actividades de los intelectuales europeos coetáneos, si bien es observable la tendencia española a manifestarse como «fruto tardío»: el Film d'Art francés precede a tareas españolas semejantes; D’Annunzio se anticipa en lo cinematográfico a Blasco Ibáñez, Zamacois, Benavente; opiniones manifestadas por los escritores europeos se encuentran posteriormente utilizadas en los artículos de los nuestros, convertidas ya en tópico de la literatura cinematográfica ${ }^{2}$.

${ }^{2}$ R. Utrera (1985), p. 16. 
A juicio de este autor, en lo que la prensa española sí resulta una adelantada es en la "valoración intelectual del Cine desde las perspectivas de la crítica cinematográfica", a través de la sección que la revista España, fundada por Ortega y Gasset en 1915, dedicó al séptimo arte y que firmaron, en distintos momentos, Federico de Onís, Martín Luis Guzmán y Alfonso Reyes. Utrera asegura que ésta se convirtió "en la primera barricada, quizás de Europa, que defiende intelectualmente al Cine. (...) Se trata de una crítica, nueva literatura, revestida de juicios literarios y comparaciones artísticas que toma como base la actualidad cinematográfica madrileña y se erige en defensora de un asunto entendido como digno de las Musas"4.

En la presentación de la película Zalacaín el aventurero (1929), de Francisco Camacho, en la tercera sesión del Cineclub Español de La Gaceta Literaria, Baroja, que había participado en el rodaje de la adaptación de su novela, leyó unas cuartillas en las que plasma sus opiniones sobre el cine y establece una clasificación de los escritores y artistas españoles en cinematófobos y cinematófilos:

El mundo literario y artístico se puede dividir, según algunos, en dos grupos: amigos del cine y enemigos del cine; cinematófilos, a un lado; cinematófobos, al otro. Los cinematófilos esperan del cine algo como el Santo Advenimiento; los cinematófobos auguran que, a fuerza de películas, iremos al caos, al abismo, a la obscuridad de la noche cineriana ${ }^{5}$.

En el lado de los "cinematófilos", que escribieron artículos periodísticos y los primeros libros de ensayo sobre el nuevo arte, podemos situar, además de los ya citados Federico de Onís, Martín Luis Guzmán y Alfonso Reyes, nombres como Manuel Machado, Azorín, Manuel Bueno, Gómez Mesa, Fernández Cuenca, Martínez de la Riva, Guillermo de Torre, Francisco Ayala, Benjamín Jarnés, César Arconada, Gómez Carrillo, Antonio Espina y Manuel Villegas López, entre otros.

Entre los grandes maestros de la época se distingue la misma diversidad con respecto al cinematógrafo: Unamuno y Antonio Machado pueden ser calificados como "cinematófobos"; Baroja, por su parte, oscila entre una postura y otra, dependiendo de la ocasión; mientras que Manuel Machado y Azorín (quien, no obstante, a partir de 1950, sintió pasión por el cine) sufrirán, con el tiempo, cierto desencanto de este arte.

También en las obras de creación de los escritores de la Edad de Plata aparecen abundantes referencias al ya considerado como séptimo arte. Véanse varios ejem-

\footnotetext{
${ }^{3}$ Ibidem, p. 17.

${ }^{4}$ p. 17.

${ }^{5}$ P. Baroja (1929), p. 1.
} 
plos $^{6}$. En Troteras y danzaderas (1913), Pérez de Ayala cuenta cómo Doña Juanita Pajares quedó "hechizada y confusa" la primera vez que asistió al cinematógrafo, pues parecía cosa de "ensalmo y brujería"7. En La señorita de Trevélez (1916) de Arniches, la mención al cine nos lleva a la identificación que sufre el espectador con los personajes y situaciones que ve en la pantalla. Flora le describe a Numeriano una escena amorosa que ha visto en una película titulada Luchando en la oscuridad e imagina que ellos pueden vivirla de una manera semejante en la vida real ${ }^{8}$. Ángela Ena, en un estudio sobre la imagen de la mujer trabajadora en la novela del primer tercio del siglo XX, recuerda el testimonio que ofrece Luisa Carnés en su obra Tea rooms: mujeres obreras (1934), en la que una joven trabajadora se empeña en imitar las actitudes y el aspecto de las grandes estrellas cinematográficas:

\begin{abstract}
se tiene por "moderna"; lo que ella llama una "chica moderna". Va sola al cinema con sus amigas y amigos, e imita los ademanes y las miradas de la "estrella" de moda. Ahora le corresponde a Marlene Dietrich. Pero Laurita está demasiado gruesa para adoptar esas difíciles aptitudes felinas de la Dietrich. En cambio, el asunto de las cejas no implica grandes trastornos; es cuestión de unas buenas pinzas y un buen lápiz. Lo verdaderamente imposible es la adopción del peinado; Laurita tiene el cabello, aunque rubio, enteramente laso, y un endemoniado flequillo que no termina nunca de crecer?
\end{abstract}

Por otra parte, son numerosos los autores que toman el mundo del cine como tema principal de sus obras, de forma que éste pasa a ser algo más que una mera referencia, como ocurre con los ejemplos que acabo de citar. Así, novelas como La mejor film: novela (1918) de Carmen de Burgos, Cinelandia: novela grande (1923) de Ramón Gómez de la Serna, La virgen de California: novela de una estrella del cinematógrafo (1925) de Julio Calvo Alfaro, Vidas de celuloide: la novela de Hollywood (1934) de Rosa Arciniega y Cinematógrafo: novela (1936) de Andrés Carranque de Ríos se centran en diferentes aspectos del ambiente cinematográfico: rodajes de películas, vidas de las estrellas, funcionamiento del aparato empresarial y publicitario de los estudios y compañías cinematográficas, pasión de los públicos por las películas y los actores, etc. De estos textos hablaré más adelante de manera específica, pues suponen un reflejo bastante completo del debate fílmico que se produce en la prensa en estas décadas.

\footnotetext{
${ }^{6}$ Para conocer algunos más, véase C. B. Morris (1993), quien examina un gran número de novelas, piezas teatrales y poemas que incorporan referencias al mundo del cine.

${ }^{7}$ R. Pérez de Ayala (1991), p. 330.

${ }^{8}$ C. Arniches (1995), p. 127.

${ }^{9}$ Á. Ena (2012), p. 148.
} 
También la narrativa breve se hace eco de estos asuntos. Basta nombrar los cuentos "La vieja del cinema", incluido en el volumen El préstamo de la difunta (1921) de Blasco Ibáñez; "Polar estrella" del libro Cazador en el alba (1930) de Francisco Ayala inspirado, al decir de la crítica, en Greta Garbo; "Los zapatos negros y el violín mudo", cuento de Concha Espina, que apareció en La Esfera el 12 de mayo de 1928 y "Una escena magistral", de Zamacois, subtitulado "cuento cinematográfico", publicado en la revista Cinegramas en 1935 y que, como explica Rafael Utrera, en este relato, "lejos de la estructura tradicional, se ofrece una narración dialogada compuesta por cinco partes que la aproxima a las fórmulas del teatro breve o al guion cinematográfico (...)"10. Unos años antes, en 1929, Baroja había publicado ya la novela breve $E l$ poeta y la princesa o El cabaret de la Cotorra Verde: novela film, en la colección $L a$ Novela de Hoy, con una técnica similar a la que emplea Zamacois en el cuento citado, tratando de imitar la estructura de un guion de cine.

El tema cinematográfico está presente también en la poesía. Sirvan de ejemplo los poemas de Alberti "Verano" de Marinero en tierra (1925), "Invierno postal" y "Carta abierta" de Cal y canto (1926-1927), "El alma en pena" de Sobre los ángeles (1929), que hace referencia al filme La Chute de la maison Usher (1928) de Jean Epstein; "Cinematógrafo" del poemario Seguro azar (1928) de Salinas; "Cinemática" del libro Ámbito (1928) de Aleixandre; "Sombras blancas" de Un río, un amor (1929) de Cernuda, inspirado en la película de Van Dyke Sombras blancas en los mares del sur (1928); o "Greta Garbo" de José María Morón, publicado en la segunda edición de su Minero de estrellas (1935).

La influencia del cine en los autores españoles de la Edad de Plata no pasa solo por la inclusión de éste como tema en sus obras, sino que es patente, en muchos de ellos, la asimilación de las técnicas propias del cine, tales como el fragmentarismo impuesto por el montaje, la visión a través de un ojo de cámara, el paso de una panorámica a un primer plano, el ritmo acelerado de la narración, la comicidad conseguida por efecto del ralentí, presentes, por ejemplo, en la expresión narrativa de Azorín y Francisco Ayala. Por otro lado, en algunos personajes de Valle Inclán se puede detectar una gestualidad cercana a la empleada por los cómicos del cine mudo y en sus obras es fundamental la presencia de elementos como la alternancia entre interiores y exteriores, el manejo de la angulación y el juego de sombras. C. B. Morris destaca la importancia de este último punto en El embrujado (1913), Luces de bohemia (1920), La rosa de papel (1924), Ligazón: auto para siluetas (1926) y La hija del capitán (1927) y afirma que

${ }^{10}$ R. Utrera (1999), p. 17. 
las sombras fascinaban a Valle-Inclán y fuera del juego de luces y sombras imaginó algunos efectos impresionantes que demuestran la fuerte impresión dejada en su mente por el desolado contraste del blanco y negro de las películas ${ }^{11}$.

Otros dramaturgos experimentaron también con el elemento filmico en sus montajes. En 1913, Muñoz Seca estrenó en el teatro Cervantes la comedia Trampa y cartón, en la que se intercaló, entre el primero y el segundo acto, la proyección de una película de Enrique Blanco, rodada por los mismos actores de la representación teatral, que servía de enlace entre ambos actos. Más tarde, haría lo mismo con otras obras. También Gregorio Martínez Sierra repetiría la experiencia en 1917, en un espectáculo presentado en el Teatro Eslava, en el que se combinaron la proyección de la película Christus (1916) de Giulio Antamoro y un montaje dramático basado en ilustraciones literarias, canciones y el auto religioso Lucero de Salvación ${ }^{12}$.

Por otra parte, hubo escritores que conocieron el cine desde dentro como guionistas, directores, actores e, incluso, como productores. Como es bien sabido, autores como Jardiel Poncela y Edgar Neville, entre otros, marcharon a trabajar a Hollywood. Muchos de ellos participaron, también, como asistentes y organizadores de los famosos cineclubs y pronunciaron conferencias sobre el cinema. A este respecto, uno de los autores más completos fue Gómez de la Serna. Además de escribir la novela, ya citada, Cinelandia: novela grande (1923), redactó varios guiones, participó como actor en Esencia de verbena (1930) de Giménez Caballero y en el cortometraje El orador, teorizó sobre el universo fílmico en diversos artículos y ensayos, hizo presentaciones de películas en el Cineclub Español, dedicó muchas de sus greguerías al cine y escribió el libreto sobre Chaplin para la ópera Charlot (1932-1933) de Salvador Bacarisse, que no llegó a representarse nunca.

Como ya queda señalado, hay una serie de novelas poco atendidas en la actualidad, como la misma Cinelandia: novela grande (1923), de Gómez de la Serna, y las ya mencionadas La mejor film: novela (1918) de Carmen de Burgos, La virgen de California: novela de una estrella del cinematógrafo (1925) de Julio Calvo Alfaro, Vidas de celuloide: la novela de Hollywood (1934) de Rosa Arciniega y Cinematógrafo: novela (1936) de Andrés Carranque de Ríos que contienen una visión bastante completa del mundo del cine de las primeras décadas del siglo XX, plasmando muchas de las opiniones que se publicaron en la prensa en torno a las distintas polémicas generadas por la aparición del cine como nuevo espectáculo. Profundizan, asimismo, en el día a día de actores, publicitarios, managers y directores que pueblan el universo hollywoodiense.

\footnotetext{
${ }^{11}$ C. B. Morris (1993), pp. 55-56.

${ }^{12}$ R. Utrera (1985), pp. 79-83; R. Gubern (1999), p. 101.
} 
Todos los textos se ocupan, en mayor o menor medida, de los mismos temas, pero no todos los abordan desde el mismo prisma estético. Gómez de la Serna se desvincula del resto por los caminos de la vanguardia. Por su parte, Rosa Arciniega, Carranque de Ríos y Carmen de Burgos despliegan su mirada crítica sobre la profesión cinematográfica desde postulados más próximos a la novela social.

Cinelandia, Vidas de celuloide y La virgen de California se adentran en el complejo entramado de Hollywood, a través del periplo de un aspirante a estrella cinematográfica, lo que les sirve para describir la convulsa vida de los habitantes de la gran "Babel loca y disparatada"13, de la ciudad "encantada" o "urbe de los milagros y las metamorfosis"14. Cinelandia o la "nueva Jerusalén", como la califica Gómez de la Serna a lo largo de su novela, es el lugar donde se puede conseguir fama y dinero, donde cualquiera puede dejar atrás una vida vulgar para convertirse en un mito.

Pero esas "vidas de celuloide", envueltas en lujo, poder y placer descontrolados esconden un lado amargo. La crítica al mercantilismo, a la banalidad y a la sensualidad vacía asoma en la mayoría de estas novelas. Hollywood es un lugar peligroso por cuanto ahoga el arte sincero y el amor auténtico. Su maquinaria, auspiciada por las estrategias de producción de grandes estrellas, fuerza a todos los que trabajan en ella a una rivalidad destructora, a la lucha constante por la ascensión o el mantenimiento en las esferas del poder cinematográfico.

Estas obras presentan a sus protagonistas como víctimas de la feroz competencia a que son sometidos por parte de los estudios de cine, que les obligan a actuar diariamente desde el egoísmo y el individualismo absolutos, lo que para ellos tiene como consecuencia un fuerte sentimiento de soledad. Jacobo Estruk, el protagonista de Cinelandia, le dice a su amante, la actriz Mary: "Se quema el alma en las películas". A lo que ella, con un cinismo que no solía tener responde: "Pues sin alma se está bien" "15. Para las grandes compañías son simples productos que se desechan cuando "caducan". En la novela de Arciniega, por ejemplo, uno de los gerentes de un estudio habla sobre "una actriz que nos envían de Francia"16 y en la de Gómez de la Serna Mary "olía a piel nueva como si acabase de ser fabricada"17.

Carranque de Ríos y Carmen de Burgos, que sitúan sus historias en España, también cargan las tintas contra los que controlan la producción de películas. En Cinematógrafo, varios personajes se esfuerzan por conseguir algún papel en una película, pues es una de las pocas vías que encuentran para intentar salir de la miseria. Los dueños de las casas cinematográficas y de las escuelas de interpretación les

\footnotetext{
${ }^{13}$ R. Arciniega (1934), p. 51.

${ }^{14}$ J. Calvo Alfaro (1925), p. 99.

${ }^{15}$ R. Gómez de la Serna (1995), p. 93.

${ }^{16}$ Ibidem, p. 19.

${ }^{17}$ p. 92 .
} 
estafan y manipulan, con el fin de que todas las ganancias sean solamente para ellos. En La mejor film, Carmen de Burgos narra cómo una compañía de artistas de cine rompe la monotonía de un pueblo catalán cuando se instala en él por unos días para el rodaje de una película. En este relato, la autora ataca la falta de profesionalidad de la compañía, lo cual recuerda las críticas de algunos escritores que, desde sus tribunas periodísticas, manifestaban su preocupación por la mala praxis de gran parte de los que trabajan en la cinematografía española en estas décadas. Martínez de la Riva alude frecuentemente a este asunto en la sección de cine de Blanco y Negro en el año $1928^{18}$. En La mejor film no se cuida ninguna de las fases de la filmación: los actores no conocen el argumento, pues el director no quiere que se sepa cuál es para evitar los plagios; el vestuario no es adecuado, así los presidiarios están caracterizados con el típico uniforme carcelario americano y los soldados, por el contrario, con trajes militares españoles; no se trabaja en la preparación del personaje, lo que da lugar a las discrepancias entre uno de los protagonistas, Alfonso Vidal, un barítono retirado que está probando suerte en el cine tras no haber logrado la fama en la ópera, y el director, a quien no le importa la labor expresiva de sus actores.

Los espectadores son también objeto de atención en estos relatos. Sienten veneración por los artistas, los imitan, quieren conocer todos los detalles de sus vidas, les escriben y les esperan a la salida del tren o de los hoteles. Imponen también, de alguna manera, su propia tiranía sobre los actores. Dice Gómez de la Serna que se ven obligados a responder a las consultas "con las que les marea el mundo"19. Este tipo de reflexiones sobre la influencia de la fama en la vida de los actores y sobre la tendencia que éstos tienen a estimular con su comportamiento excéntrico el interés obsesivo del público las expone el ya mencionado crítico Ramón Martínez de la Riva en algunos artículos publicados en la revista Blanco y Negro: El funambulismo de los artistas de «cine» (11-11-1928), La «vedette» de los grandes «sucesos» (239-1928), Los actores tal como son (16-9-1928) o "Charlot" y la película de su vida (18-3-1928). También Gómez de la Serna se burla de las excentricidades de los artistas, como cuando presenta al personaje de Elsa, que acaba de echarse la siesta en un armario $^{20}$.

En estas novelas se insiste, igualmente, en la fascinación que suscitan las estrellas de cine, con sus trajes caros, mansiones lujosas, tertulias, fiestas y bailes elegantes y, por supuesto, su belleza. En todas se pueden encontrar alusiones explícitas a numerosos actores de la época como Greta Garbo, Adolphe Menjou, Emil Jan-

\footnotetext{
${ }^{18}$ De este tema me he ocupado en Cine y novela cinematográfica en la revista Blanco y Negro (1928). Puede consultarse en http://eprints.ucm.es/13992/.

${ }^{19}$ R. Gómez de la Serna (1995), p. 40.

${ }^{20}$ Ibidem, p. 44.
} 
nings, Francesca Bertini, los Barrymore, Marlene Dietrich, o referencias veladas como en Cinelandia, donde el personaje de Mary, que representa el prototipo de la jovencita ingenua, esconde a la actriz Mary Pickford. Sin duda, de entre todos ellos, destaca la figura de Chaplin, cuya presencia es muy recurrente en estas obras. Sobre él escriben prácticamente todos los autores que tratan el tema del cine, tanto en prensa como en libros de ensayo. Valga el ejemplo de César Arconada y sus libros Vida de Greta Garbo (1929) y 3 cómicos del cine (1931) sobre Chaplin, Harold Lloyd y Clara Bow.

También Azorín se ocupa de los actores en numerosos artículos. En ellos comenta la interpretación actoral, la voz, el tono y la belleza de las actrices. Este último rasgo es el más repetido cuando se alude a las artistas en las novelas analizadas. Calvo Alfaro critica en su novela el hecho de que el atractivo físico de las estrellas femeninas es el único requisito que les piden los dueños de las productoras a la hora de darles un papel. Es elocuente el anuncio que una de estas inserta en el periódico para encontrar aspirantes: "Se necesita señoritas hermosas de rostro y cuerpo perfecto. Es indiferente el color del cabello, la estatura y la educación. Han de actuar en el elenco artístico de la compañía «Norma», productora de películas cinematográficas." 21 .

Otro de los juicios negativos frecuentes en estos textos es el ansia de actores y directores por alcanzar la gloria rápidamente, con poco esfuerzo, el deseo de una mayor fama y dinero, la complacencia en el fracaso de los compañeros de profesión, que suponen siempre una amenaza al éxito propio, la búsqueda permanente del placer así como el engaño, la mentira y la deslealtad.

Hollywood goza de una total libertad sexual y esto se convierte en auténtica obsesión crítica en la obra de Calvo Alfaro, que advierte constantemente del peligro que supone esta ciudad, amenaza constante de la honra de todas las mujeres. Huelga decir que sobre la libertad sexual masculina no hace ningún comentario.

Por el contrario, el tratamiento que Gómez de la Serna hace del erotismo y la sexualidad en su Cinelandia no revela reprobación alguna, sino más bien al contrario. El sexo o, por mejor decir, el liberalismo en este aspecto de la vida hollywoodiense tiene, de hecho, un peso fundamental en su novela.

Por su parte, los reproches que se detectan en la novela de Arciniega no parecen una acusación directa contra esa sexualidad abierta que se practica en el ámbito artístico. Lo que sí recrimina la autora es la infidelidad y la prevalencia del simple placer sobre el amor verdadero. El protagonista de Vidas de celuloide, Eric Freyer, llega a Hollywood para convertirse en un actor famoso y engaña a su mujer, a la que ha dejado en Berlín y con la que ha compartido pobreza e ilusiones de una vida

${ }^{21}$ J. Calvo Alfaro (1925), p. 96. 
mejor. La olvida en el momento en que consigue fama y fortuna y la sustituye por una actriz de gran renombre y belleza, Olga D'Anti. Su interés por ella se relaciona con el deseo de conquista de una estrella, inaccesible para la mayoría, y con el orgullo que supone llevarla de su brazo ante todo el mundo, provocando así la envidia de todos. La otra cara de la crítica de Arciniega la ocupa la repulsión que le produce el hecho de que Henriette, la esposa de Freyer, que se dedica al teatro de variedades, se vea siempre acosada por los empresarios para los que trabaja. Cuando ella no se presta a sus proposiciones la despiden. "Su pobreza es siempre el blanco escogido para herirla" 22 .

La visión poco favorecedora del ambiente de la gran urbe cinematográfica, materializada en la decadencia de costumbres y valores de los actores y los personajes de las películas, queda reflejada en la prensa por autores como Unamuno, quien censura la ligereza de ropa de las protagonistas femeninas y el influjo pernicioso del cine sobre la juventud; o Ramiro de Maeztu, que toma "claras posiciones anticinematográficas más en función de sus efectos que de su esencia"23. Es decir, no cuestiona tanto el fondo artístico o estético del cine, sino su influencia negativa en la sociedad. En esta línea se situaron también Concha Espina y Palacio Valdés, lo que no impidió que aceptaran la adaptación cinematográfica de varias de sus obras. Baroja, por su parte, simplemente concibe el cine como modificador de la conducta y las costumbres.

Uno de los puntos en los que más se insiste en las novelas analizadas es la falsedad del mundo cinematográfico. La ficción lo invade todo. Hasta el modo de conducirse de los actores, cuando no están interpretando, está traspasado de un carácter inequívocamente fílmico. Cinelandia es, de entre estas, la obra que más destaca, en este sentido, por la cantidad de alusiones a la falsedad del cine y a la ficcionalización de la vida real de los personajes. A este respecto comenta Ricardo Fernández Romero:

A través de las páginas de Cinelandia pueden comprobarse los múltiples puntos de encuentro entre el cine y la literatura de Ramón. De todos ellos ofrece gran interés la reflexión de Ramón sobre el cine como arte esencialmente artificioso, quizá el más artificioso de todos al ser sólo luz y sombras fugaces proyectadas

\footnotetext{
${ }^{22}$ R. Arciniega (1934), p. 42.

${ }^{23}$ R. Utrera (1981), p. 89.
} 
sobre una pantalla. A este arte se adecua la novela "fingida" 24 , como diría Francisco Umbral, que es Cinelandia ${ }^{25}$.

La ciudad es falsa, las tiendas parecen una falsificación, se habla de la falsa Cléo de Mérode, del falso film, de los falsos viejos de los films y de los falsos doctores -en una fiesta, cuando una actriz pide a gritos un doctor, "Erbert, como impulsado por un deber cinematográfico" 26 acude en su ayuda-. No solo ellos se creen el rol que interpretan habitualmente, sino también un actor que se parece al asesino Ravarol, que cuenta a todo el que le pregunta las historias de sus crímenes como si fueran ciertos. O Teodoro Bouvel, quien había hecho en una película de padre de Carlota Bray, "la menor que hace que hombres de todas partes deseen y fuercen a niñas"27, y ahora la quiere como un padre y ella le llama padre, deseosa de que la proteja ${ }^{28}$. El gran magnate de Cinelandia finge que está terminando de escribir una carta ante los aspirantes que llegan a su despacho ${ }^{29}$. Es decir, la apariencia es el gran valor en esta ciudad. Se habla también de lágrimas falsas, de falsos toreros, que "viven como toreros, pero sin sus peligros" 30 , de joyas falsas, "las de verdad son muy sosas en el cinematógrafo"31, de pestañas falsas, del "beso más falso del mundo" ${ }^{\text {, }}$, etc.

En Cinelandia, la semana está compuesta por seis falsos domingos y uno verdadero $^{33}$, las actrices "tienen gestos de hipocresía sentimental" y "están tan locas como las locas verdaderas" 34 y sus habitantes "desdeñan el Duomo real por adoración al suyo imitado" 35 . Al manicomio lo llaman "Museo de la expresión" y el lago donde "cisnes y mujeres bogaban juntos" es artificial ${ }^{36}$. Carlota, la joven actriz de moda, “¡sabía tan bien hacer que amaba!”37 y su compañera de profesión, conocida

${ }^{24}$ Con este término, Umbral se refiere a la organización narrativa de algunas novelas de Gómez de la Serna, en las que tiene más importancia la concatenación de metáforas brillantes que el argumento novelesco, lo cual las aleja de la estructura tradicional.

${ }^{25}$ R. Fernández Romero (1996).

${ }^{26}$ R. Gómez de la Serna (1995), p. 214.

${ }^{27}$ Ibidem, p. 165.

${ }^{28}$ p. 166.

${ }^{29}$ p. 53.

${ }_{30}$ p. 147.

31 p. 159.

32 p. 175.

${ }_{33}$ p. 211.

${ }^{34}$ pp. $95-96$.

35 p. 179.

${ }_{37}^{36}$ p. 90 .

37 p. 210. 
como Venus de Plata, "tira el sombrero con aire cinematográfico"38 al entrar en su casa. Dos grandes estrellas como Elsa y Max marchan a una localidad cercana porque "tenían ansiedad por pasar por un pueblo verdadero" y, después de comparar su vida con la de los "seres reales", vuelven a Cinelandia", donde los artistas "pierden el nombre y adquieren el nombre cinematográfico" 40 . En el rodaje de una película, Carlota "entregaba una mentira llena de seriedad. Era la mujer de la que no se puede dudar y, sin embargo, estaba creando la mentira mayor del mundo, la mentira de una película. Parecía una verdad su actuación. Tenía gestos de guardar de verdad en su corazón las carantoñas de aquel hombre. No era una actriz aquella mujer. Era una mujer verdadera de brazos sinceros, de belleza real, pudibunda, austera" ${ }^{91}$.

Para Rosa Arciniega, la atmósfera ficcional que envuelve las "vidas de celuloide" de los personajes de su novela tiene primordialmente un carácter negativo. Cuando Eric Freyer llega a Hollywood descubre que "todas las construcciones, paisajes y países están allí imitados. La sensación de su autenticidad era perfecta. Pero al transponerlas aparece toda su desoladora falsedad, un fondo para encuadrar la farsa" ${ }^{42}$. Maurice Roger, que ha servido a Freyer de guía por la gran urbe le dice en una ocasión:

No sabe usted nada de esto, amigo Freyer. Nada. Como no lo sabe tampoco el mundo de los aficionados, ni aun el noventa por ciento de los que viven aquí. La vida de estos estudios es un misterio, del cual sólo se conoce lo que conviene a la alta dirección. ¡El día que usted pueda penetrar en la verdadera existencia de esos millones de hombres y mujeres que se mueven ahí, bajo la falsa luz de los soles artificiales! !El día que usted sepa lo que hay detrás de todos esos mentidos oropeles que deslumbran al mundo!... En fin, amigo Eric; prepárese usted a cambiar de piel como las serpientes. Y mienta, mienta usted estrepitosamente. Por muy inverosímiles que sean sus mentiras siempre parecerán cuentos ingenuos al lado de los que aquí escuchará usted”33.

Aunque ambos autores tratan esta cuestión, la perspectiva desde la que se ocupan del tema es bien distinta. Si para Rosa Arciniega el ambiente hollywoodiense y la vida de sus actores están marcados por la falsedad, para Gómez de la Serna se trata de un revestimiento ficcional sin connotaciones negativas. Como señala Fernández Romero, "Cinelandia es la ciudad falsa, es decir artificiosa, esencialmente artística,

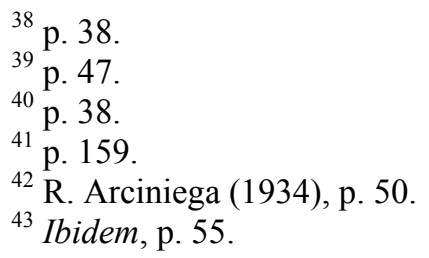


al modo como entiende el arte Ramón Gómez de la Serna"44. Para él, la urbe del cinema es el universo de la ficción, del arte y de la invención. Es un lugar libre y disparatado.

Quizá la crítica más significativa que hace Gómez de la Serna en su novela es la dirigida contra las malas películas, es decir, contra las películas comerciales, mayoritarias, que repiten los mismos esquemas y que no se distinguen por su calidad estética, tema presente igualmente en las obras de Carmen de Burgos y Carranque de Ríos, pero en relación con el cine español, y en varios artículos periodísticos de Manuel Machado y Azorín. Las contrapone a las "películas de ensayo", a las que considera "películas poéticas", aquellas que "están regidas por el verso" ${ }^{45}$ :

Estos escritores de cinematógrafo de ensayo están apartados de los que triunfan en el cine actual y que son, más que escritores, confeccionadores de una cosa indefinida, tanto que para encontrar el pensamiento que se niega a brotar en ellos necesitan buscar incitaciones diferentes y extrañas (...), buscando la inspiración baja, fehaciente, digna de las burguesías y las clases medias que van al cine ${ }^{46}$.

En la prensa, tanto general como especializada, los escritores de la Edad de Plata escribieron sobre otros temas que, aunque con menor desarrollo, están presentes también en estas obras: el cine y su relación con el sueño y con las ansias de eternidad, la comparación con el teatro, el paso del cine mudo al "parlante", las aportaciones de la gestualidad y del dinamismo cinematográficos, el vínculo entre el $\mathrm{ci}$ nema y la modernidad, su capacidad para convertirse en un arte de masas o la discusión sobre si el cine es un arte o no.

Como puede verse, las novelas estudiadas resultan de importancia capital para comprender la visión que los escritores españoles del primer tercio del siglo XX tenían sobre Hollywood, sobre la vida de los artistas y sobre el cine entendido como arte y espectáculo. Con sus obras contribuyeron, desde el ámbito de la creación literaria, a enriquecer el debate cinematográfico de aquellas décadas.

\footnotetext{
${ }^{44}$ R. Fernández Romero (1996).

${ }^{45}$ R. Gómez de la Serna (1995), p. 136.

${ }^{46}$ Ibidem, pp. 137-138.
} 


\section{OBRAS CITADAS}

ARCINIEGA, Rosa: Vidas de celuloide: la novela de Hollywood, Madrid, Cenit, 1934.

ARNICHES, Carlos: La señorita de Trevélez, ed. de Andrés Amorós, Madrid, Cátedra, 1995.

BAROJA, Pío: El poeta y la princesa o El cabaret de la Cotorra Verde: novela film, ilustr. de Puyol, La Novela de Hoy, 398 (1929).

Burgos, Carmen de: La mejor film: novela, La Novela Corta, 155 (1918).

CAlvo Alfaro, Julio: La Virgen de California: novela de una estrella del cinematógrafo, Barcelona, Mediterráneo, 1925.

CARRANQUe De Ríos, Andrés: Cinematógrafo, introd. de Antonio Muñoz Molina, Madrid, Viamonte, 1997.

ENA BORDONADA, Ángela: "Imagen de la mujer trabajadora en la novela del primer tercio del s. XX: Tea Rooms. Mujeres obreras, de Luisa Carnés", en Nuevos modelos: cultura, moda y literatura (España 1900-1939), ed. de Margherita Bernard e Ivana Rota, Bergamo, Bergamo University Press-Sestante Edizioni, 2012.

FERNÁNDEZ Romero, Ricardo: "Cine y literatura en Cinelandia, de Ramón Gómez de la Serna", Especulo, 4 (1996).

GÓMEZ DE LA SERNA, Ramón: Cinelandia, introd. de Francisco Gutiérrez Carbajo, Madrid, Valdemar, 1995.

GuBERN, Román: Proyector de luna: la generación del 27 y el cine, Barcelona, Anagrama, 1999.

"Palabras de Pío Baroja", La Gaceta Literaria, 53 (1-3-1929).

MORRIS, Cyril Brian: La acogedora oscuridad: el cine y los escritores españoles (1920-1936), Córdoba, Filmoteca de Andalucía, 1993.

PÉREZ DE AYALA, Ramón: Troteras y danzaderas, ed. de Andrés Amorós, Madrid, Castalia, 1991.

UTRERA, Rafael: Modernismo y 98 frente a cinematógrafo, Sevilla, Secretariado de Publicaciones de la Universidad, 1981.

-: Escritores y cinema en España: un acercamiento histórico, pról. de Jorge Urrutia, Madrid, Ediciones J.C, 1985.

: Cuentos de cine: de Baroja a Buñuel, selecc., pról. y ed. de Rafael Utrera, ilustr. de Marina Arespacochaga, Madrid, Clan, 1999. 